\title{
Electroosmotic flow of a power-law fluid in a non-uniform microchannel
}

\author{
Chiu-On Ng, ${ }^{1}$ Cheng Qi \\ Department of Mechanical Engineering, The University of Hong Kong, \\ Pokfulam Road, Hong Kong
}

January 13, 2015

\begin{abstract}
An analytical model is presented for electrokinetic flow of a power-law fluid through a slit channel with gradually varying channel height and wall potential. With the nearwall depletion effect taken into account, the present model is based on the lubrication approximation and the use of the Helmholtz-Smoluchowski slip boundary condition. It is found that interaction between the wall undulation and the wall potential modulation, under the combined action of hydrodynamic and electric forcings, may give rise to a rich set of nonlinear behaviors for flow of a non-Newtonian fluid in the channel. In particular, the linear superposition of flow components due separately to the two forcings is found to work only for a strictly uniform channel; non-uniformity in channel height or wall potential distribution will spoil such linearity.
\end{abstract}

Keywords: electroosmotic flow; power-law fluid; depletion layer; Helmholtz-Smoluchowski slip.

\footnotetext{
${ }^{1}$ Corresponding author. Tel:(852) 2859 2622; Fax: (852) 28585415; E-mail address: cong@hku.hk (C.-O. $\mathrm{Ng})$.
} 


\section{Introduction}

Electroosmosis, in which a flow is driven by an applied electric field, is now commonly used to generate flow in a microchannel. This process draws upon the electrokinetics of the free ions in the electric double layer (EDL) that is formed near a charged surface. Electroosmotic (EO) flow is known to offer several advantages over pressure-driven flow: a more precise flow control, a much flatter velocity profile, and a flow rate that is less dependent on the channel size. Studies on EO flow of Newtonian fluids abound in the literature. In contrast, EO flow of non-Newtonian fluids has not received much attention until recent years, as driven by the need to handle complex biological fluids in microfluidics.

Thus far, the power-law model has been the most chosen rheological model by various studies, analytical or numerical, on EO flow of non-Newtonian fluids. Examples include Chakraborty [1], Berli and Olivares [2], Zhao et al. [3], Bharti et al. [4], Olivares et al. [5], Tang et al. [6], Zhao and Yang [7-9], Berli [10], Vasu and De [11,12], Babaie et al. [13], Hadigol et al. [14], Sadeghi et al. [15], Cho et al. [16,17], Deng et al. [18], Shamshiri et al. [19], Vakili et al. [20], and Zhu et al. [21]. Despite these numerous studies, the nonlinear interplay between the hydrodynamic and electric forcings in driving a power-law fluid through a non-uniform channel is yet to be elucidated.

A non-Newtonian fluid is one that exhibits nonlinear rheological behaviors, which will cause a problem of non-Newtonian fluid flow not amenable to analytical analysis in general. The only exception is when the fluid is subjected to simple shear, for which the stress can be determined prior to the velocity. Existing analytical work (e.g., Berli and Olivares [2], Zhao et al. [3], Das and Chakraborty [22], $\mathrm{Ng}$ and Qi [23]) on EO flow of a power-law fluid have all considered simple geometries: flow over a flat surface, or through a uniform parallel-plate or circular channel.

Another concern is the linearity of the relationship between the driving forces and the flow rate, which is normally satisfied by Newtonian fluids. The so-called Onsager relations are expected not to hold for non-Newtonian fluids owing to the nonlinear fluid rheology. Nevertheless, when wall depletion effects are taken into account, EO flow of non-Newtonian fluid has been shown by Berli and Olivares [2] to be a linear combination of the components 
due separately to the hydrodynamic and electric forcings. They found that nonlinear effects are limited to the pressure-driven component of the flow, and the Onsager reciprocity (i.e., equality of the non-conjugate streaming coefficients) is satisfied. They have, however, considered strictly one-dimensional flow in uniform microchannels only. Whether such linearity applies to non-unidirectional flow remains an unanswered question.

This paper aims to look into combined pressure-driven and electroosmotic flow of a powerlaw fluid in a non-uniform slit channel. The objective is to find out how the hydrodynamic and electric forcings may interact with each other in driving non-parallel flow of a power-law fluid through a channel with axial non-uniformities. The channel height as well as the wall charge may change as a function of axial position. We shall show that, for non-parallel flow, linear superposition of components due to the two forcings does not work for a non-Newtonian fluid, even when a Newtonian depletion layer is taken into account.

The rationale for considering wall depletion effects for EO flow of non-Newtonian fluids in microchannels has been explained in detail by Berli and Olivares [2]. The depletion or skimming layer (e.g., Barnes [24], Tuinier and Taniguchi [25]) is a thin layer near a solid wall in which the fluid is depleted of the macromolecules that make up the non-Newtonian behavior of the bulk fluid. Therefore, in this region, the fluid is essentially the Newtonian solvent. This layer is inaccessible to the center of the macromolecules, and therefore has a thickness comparable to the radius of gyration of the macromolecules. As estimated by Berli and Olivares [2], this layer can be thick enough to cover the EDL, which will hence confine the electrokinetic driving force to a region where the fluid is Newtonian. The bulk non-Newtonian fluid is under the hydrodynamic driving force only. By this approach, Berli and Olivares [2] showed that the streaming conductance depends on the Newtonian solvent viscosity only, and is independent of the non-Newtonian rheology of the bulk fluid. Onsager reciprocity is thereby achieved as if the fluid were entirely Newtonian. Other authors who also considered a depletion layer in their studies on EO flow of non-Newtonian fluids include, among others, Olivares et al. [5], Berli [10], and Zimmerman et al. [26]. The issue of possible nonlinear interplay between the hydrodynamic and electric forcings for a non-parallel flow is, however, not addressed in these studies.

In addition to taking into account the depletion effects, the present problem is simplified 
by means of two approximations, which enable the problem to be solved as analytical as possible. Also, a simplified problem will allow the physics to be revealed without involving too much mathematics. First, the lubrication approximation is adopted. This approximation is valid as long as the Reynolds number is sufficiently low and there exists a sharp contrast in length scales. The ratio of the length scale for variations in the axial direction to that in the transverse direction needs to be much smaller than unity. This ratio times the Reynolds number has to be very small as well. These two conditions will enable the problem to be solved in a quasi-parallel manner, as the axial velocity will be much larger in magnitude than the transverse velocity. In this problem, the channel height and the wall potential are assumed to change slowly with axial position, over a length scale much greater than the channel height. For Newtonian EO flow, the lubrication approximation has been applied previously by, among others, Ajdari [27,28], Long et al. [29], Ghosal [30], and Ng and Zhou [31,32]. In particular, Ajdari [27] looked into superposition of periodic surface charge and topography patterns. For flow between two parallel plates, he showed that the combined effect of charge and shape modulation on the surfaces is to generate net flow even when the plates are on average electro-neutral. A similar configuration of wall charge and shape modulation is considered in the present study, but for a non-Newtonian EO flow. Second, following MacInnes et al. [33], we shall not resolve the EDL directly for the electrokinetic pumping, but will adopt the Helmholtz-Smoluchowski (HS) slip boundary condition instead [26]. The conditions for this approximation, as have been discussed by MacInnes [34], are assumed to be valid in the present study. Here, we assume that the channel height, which is of the order of $100 \mu \mathrm{m}$, is much larger than the depletion layer thickness $(\sim 100 \mathrm{~nm})$, which is in turn larger than the EDL thickness $(\sim 10 \mathrm{~nm})$. Without directly involving the depletion layer, the analysis can be performed in a simplified manner focusing on the bulk fluid flow only.

\section{The Problem}

The present problem is to consider steady pressure-driven and electroosmotic flow of a powerlaw fluid through a slit microchannel, where the channel height as well as the wall potential may vary slowly and periodically with axial position. Figure 1 shows a definition sketch of 
our problem, where $(x, y)$ are the axial and transverse coordinates, and the $x$-axis is along the centerline of the channel. Only axisymmetric flow is considered: the two walls are at $y= \pm h(x)$ and the wall potential is given by $\zeta=\zeta(x)$, both being periodic functions of $x$ with the same wavelength $L$. The wavelength $L$, which is the length scale for variations of flow in the axial direction, is assumed to be much longer than the channel height: $L \gg h$. With this sharp contrast in length scales, we further assume that the Reynolds number of the flow is so small that the lubrication approximation can be applied here.

The bulk fluid is a non-Newtonian fluid that can be modeled as a power-law fluid. The walls are assumed to be non-adsorbing, and therefore very near a wall is a depletion layer, where the fluid, owing to the absence of the inclusion, is much less viscous than the bulk fluid and can be taken as Newtonian. It is also assumed that the electric double layer is thinner than the depletion layer so that the electrokinetic effect is confined to the region of Newtonian fluid adjacent to the wall. We further assume that the depletion layer thickness is much smaller than the channel height. As a result, the electrokinetic forcing reduces to a Helmholtz-Smoluchowski (HS) slip velocity:

$$
u=u_{s}(x)=-\frac{\epsilon E_{x}}{\mu_{s}} \zeta(x) \quad \text { at } y= \pm h(x)
$$

where $u$ is the axial velocity, $\epsilon$ and $\mu_{s}$ are the permittivity and dynamic viscosity of the solvent in the depletion layer, and $E_{x}$ is the applied axial electric field. By virtue of the gentle change in the wall shape, the wall steepness is very small, and the HS slip condition can be applied as if the walls were flat.

On invoking the lubrication approximation, the Cauchy momentum equation is simplified to

$$
0=-\frac{\partial p}{\partial x}+\frac{\partial \tau}{\partial y}
$$

where $p=p(x)$ is the pressure, and $\tau=\tau(x, y)$ is the shear stress. The pressure here is composed of applied pressure and pressure induced by non-uniformity of the channel.

On denoting the pressure gradient by $K(x)=-\partial p / \partial x$, the equation above gives a linear stress distribution across the channel

$$
\tau=-K y
$$


where the symmetry condition $\tau(y=0)=0$ is used. By symmetry about the centerline, it suffices for us to consider flow in the upper half of the channel: $0 \leq y \leq h(x)$.

Under simple shear, a power-law fluid will have the following rheological behavior

$$
\tau=\left\{\begin{array}{ll}
\mu\left(\frac{\mathrm{d} u}{\mathrm{~d} y}\right)^{n} & \text { for } \tau>0, \frac{\mathrm{d} u}{\mathrm{~d} y}>0 \\
-\mu\left(-\frac{\mathrm{d} u}{\mathrm{~d} y}\right)^{n} & \text { for } \tau<0, \frac{\mathrm{d} u}{\mathrm{~d} y}<0
\end{array},\right.
$$

where $\mu$ is the consistency, and $n$ is the power-law index of the fluid. The shear-thinning, Newtonian, and shear-thickening behaviors are exhibited when $n<1,=1,>1$, respectively. We need to distinguish between the cases where the stress is positive or negative when Eq. (4) is substituted into Eq. (3).

When $K>0$ (i.e., under favorable pressure gradient), the stress is negative in the upper half of the channel. The axial velocity is readily found to be

$$
u=u_{s}+\left(\frac{K}{\mu}\right)^{\frac{1}{n}}\left(\frac{n}{1+n}\right)\left[h^{\frac{1+n}{n}}-y^{\frac{1+n}{n}}\right] \quad \text { for } 0 \leq y \leq h(x),
$$

where the HS slip condition (1) is used. The volume flow rate through the channel is then given by

$$
q=2 \int_{0}^{h} u \mathrm{~d} y=2 u_{s} h+2\left(\frac{K}{\mu}\right)^{\frac{1}{n}}\left(\frac{n}{1+2 n}\right) h^{\frac{1+2 n}{n}} .
$$

By continuity, $q$ is independent of axial position.

When $K<0$ (i.e., under adverse pressure gradient), the stress is positive in the upper half of the channel. In this case, the axial velocity and flow rate are found to be

$$
\begin{gathered}
u=u_{s}-\left(-\frac{K}{\mu}\right)^{\frac{1}{n}}\left(\frac{n}{1+n}\right)\left[h^{\frac{1+n}{n}}-y^{\frac{1+n}{n}}\right] \quad \text { for } 0 \leq y \leq h(x), \\
q=2 u_{s} h-2\left(-\frac{K}{\mu}\right)^{\frac{1}{n}}\left(\frac{n}{1+2 n}\right) h^{\frac{1+2 n}{n}} .
\end{gathered}
$$

From Eqs. (6) and (8), it is clear that $K(x)>0$ where $q>2 u_{s}(x) h(x)$, and $K(x)<0$ where $q<2 u_{s}(x) h(x)$. On rearranging terms, a single expression, which is valid for any $K$, is obtainable for the pressure gradient and the axial velocity as a function of the flow rate:

$$
K=\mu\left(\frac{1+2 n}{2 n}\right)^{n} \frac{\left|q-2 u_{s} h\right|^{n-1}}{h^{1+2 n}}\left(q-2 u_{s} h\right),
$$




$$
u=u_{s}+\left(\frac{1+2 n}{1+n}\right) \frac{\left(q-2 u_{s} h\right)}{2 h}\left[1-\left(\frac{y}{h}\right)^{\frac{1+n}{n}}\right] .
$$

Integrating the pressure gradient over one wavelength gives

$$
\begin{aligned}
\int_{0}^{L} K \mathrm{~d} x & =p(0)-p(L) \equiv \Delta P \\
& =\mu\left(\frac{1+2 n}{2 n}\right)^{n} \int_{0}^{L}\left(q-2 u_{s} h\right) \frac{\left|q-2 u_{s} h\right|^{n-1}}{h^{1+2 n}} \mathrm{~d} x
\end{aligned}
$$

where $\Delta P$ is net pressure drop in one wavelength, which is dictated by the applied pressure forcing. The equation above is the one by which the flow rate $q$ can be solved for.

Let us now introduce the following normalized variables (distinguished by overhead carets):

$$
\left.\begin{array}{r}
\hat{x}=x / L, \quad(\hat{y}, \hat{h})=(y, h) / h_{0}, \quad\left(\hat{u}, \hat{u}_{s}\right)=\left(u, u_{s}\right) / u_{0}, \\
\hat{q}=q /\left(2 u_{0} h_{0}\right), \quad \hat{K}=K /\left(\mu u_{0}^{n} / h_{0}^{1+n}\right), \quad \Delta \hat{P}=\Delta P /\left(\mu u_{0}^{n} L / h_{0}^{1+n}\right)
\end{array}\right\},
$$

where $u_{0}$ and $h_{0}$ are the characteristic scales for the axial velocity and the channel height, respectively. In terms of these dimensionless variables, the present problem consists of the following key equations. First, for given $\Delta \hat{P}, \hat{u}_{s}(\hat{x})$ and $\hat{h}(\hat{x})$, the flow rate $\hat{q}$ is determined from

$$
\left(\frac{1+2 n}{n}\right)^{n} \int_{0}^{1}\left(\hat{q}-\hat{u}_{s} \hat{h}\right) \frac{\left|\hat{q}-\hat{u}_{s} \hat{h}\right|^{n-1}}{\hat{h}^{1+2 n}} \mathrm{~d} \hat{x}=\Delta \hat{P} .
$$

Then, the pressure gradient $\hat{K}(\hat{x})$ and axial velocity $\hat{u}(\hat{x}, \hat{y})$ are given by

$$
\begin{gathered}
\hat{K}=\left(\frac{1+2 n}{n}\right)^{n} \frac{\left|\hat{q}-\hat{u}_{s} \hat{h}\right|^{n-1}}{\hat{h}^{1+2 n}}\left(\hat{q}-\hat{u}_{s} \hat{h}\right), \\
\hat{u}=\hat{u}_{s}+\left(\frac{1+2 n}{1+n}\right) \frac{\left(\hat{q}-\hat{u}_{s} \hat{h}\right)}{\hat{h}}\left[1-\left(\frac{\hat{y}}{\hat{h}}\right)^{\frac{1+n}{n}}\right] \quad \text { for } 0 \leq \hat{y} \leq \hat{h}(\hat{x}) .
\end{gathered}
$$

In the Newtonian limit $(n=1)$, Eq. (13) can be solved straightforwardly to give

$$
\hat{q}_{n=1}=\left[\frac{\Delta \hat{P}}{3}+\left\langle\hat{u}_{s} \hat{h}^{-2}\right\rangle\right]\left\langle\hat{h}^{-3}\right\rangle^{-1},
$$

where the angle brackets denote averaging over one wavelength:

$$
\langle f\rangle \equiv \int_{0}^{1} f \mathrm{~d} \hat{x} .
$$

In the special case where $\hat{h}$ and $\hat{u}_{s}$ are both constants (i.e., uniform channel), Eq. (13) can also be solved easily to give

$$
\hat{q}_{\text {unifm }}= \pm\left(\frac{n}{1+2 n}\right) \hat{h}^{\frac{1+2 n}{n}}( \pm \Delta \hat{P})^{\frac{1}{n}}+\hat{h} \hat{u}_{s}
$$


where the upper/lower signs are for $\Delta \hat{P}>0,<0$, respectively.

In general, Eq. (13) has to be solved numerically. An efficient iterative solution scheme can be applied here. Based on the $i$ th trial flow rate $\hat{q}^{(i)}$, the left hand side of Eq. (13) is

evaluated to give the $i$ th trial pressure drop $\Delta \hat{P}^{(i)}$, which is to compare with the given $\Delta \hat{P}$. The difference of the two will determine the $(i+1)$ th trial flow rate as follows:

$$
\hat{q}^{(i+1)}=\hat{q}^{(i)}+\frac{\Delta \hat{P}-\Delta \hat{P}^{(i)}}{(\mathrm{d} \Delta \hat{P} / \mathrm{d} \hat{q})^{(i)}}
$$

where

$$
\frac{\mathrm{d} \Delta \hat{P}}{\mathrm{~d} \hat{q}}=\left(\frac{1+2 n}{n}\right)^{n} n \int_{0}^{1} \frac{\left|\hat{q}-\hat{u}_{s} \hat{h}\right|^{n-1}}{\hat{h}^{1+2 n}} \mathrm{~d} \hat{x} .
$$

The Newtonian $\hat{q}_{n=1}$ given by Eq. (16) can be used as the initial guess $\hat{q}^{(1)}$. Normally, convergence to the solution can be achieved in 5 iterations, where the accuracy of the solution depends on that of the numerical evaluation of the definite integrals.

\section{Discussion}

We shall from here on for simplicity omit the overhead carets in our notation. Equations (13)-(16) are valid for any $h$ and $u_{s}$ as long as they are slow functions of $x$. Here, let us introduce the following sinusoidal functions for the channel height and HS slip velocity:

$$
\begin{gathered}
h(x)=1+h^{\prime} \cos (2 \pi x), \\
u_{s}(x)=\bar{u}_{s}+u_{s}^{\prime} \cos (2 \pi x+\phi),
\end{gathered}
$$

where $\bar{u}_{s}$ is the mean HS slip velocity, $h^{\prime}$ and $u_{s}^{\prime}$ are the amplitudes of the wall shape and slip modulation, respectively, and $\phi$ is a phase shift between the two modulation waves.

With Eqs. (21) and (22), the Newtonian limit is now given by

$$
q_{n=1}=\frac{2\left(1-h^{\prime 2}\right)^{5 / 2}}{3\left(2+h^{\prime 2}\right)} \Delta P+\frac{2\left(1-h^{\prime 2}\right)}{2+h^{\prime 2}}\left(\bar{u}_{s}-h^{\prime} u_{s}^{\prime} \cos \phi\right),
$$

while for the special case of a uniform channel $\left(h^{\prime}=u_{s}^{\prime}=0\right)$,

$$
q_{\text {unifm }}= \pm\left(\frac{n}{1+2 n}\right)( \pm \Delta P)^{\frac{1}{n}}+\bar{u}_{s}
$$


where the plus/minus sign is used when $\Delta P$ is positive/negative, respectively. Obviously, in either of these two particular cases (Newtonian limit or uniform channel), the flow is a linear combination of the hydrodynamic and electrokinetic effects. There is essentially no coupling between the two forcings. Another special case is also noteworthy. When $\Delta P=0$ and $h^{\prime}=0$ (i.e., flat walls),

$$
q=\bar{u}_{s} \quad \text { for any } n \text { and } u_{s}^{\prime} \text {. }
$$

These limiting and special cases will be further examined in our numerical discussion below. The computation requires 6 input parameters: $n, \Delta P, h^{\prime}, \bar{u}_{s}, u_{s}^{\prime}$ and $\phi$. We have chosen Simpson's formula to evaluate the definite integrals in Eqs. (13) and (20).

Let us first look into the issue of linear superposition of the hydrodynamic and electrokinetic effects. In this regard, we define the following types of flow rate:

$$
\begin{aligned}
q_{\mathrm{PO}} & =\text { rate of flow due to hydrodynamic forcing }(\Delta P) \text { only; } \\
q_{\mathrm{EO}} & =\text { rate of flow due to electric forcing }\left(u_{s}\right) \text { only; } \\
q_{\mathrm{comb}} & =\text { rate of flow due to combined action of } \Delta P \text { and } u_{s} .
\end{aligned}
$$

Figure 2 shows these flow rates as a function of the phase, for $n=0.5,1,1.5, \Delta P=1$, $h^{\prime}=0.5, \bar{u}_{s}=0$ and $u_{s}^{\prime}=1$. Clearly, except for $n=1$, the sum of $q_{\mathrm{PO}}$ and $q_{\mathrm{EO}}$ (represented by the dashed lines) is not equal to $q_{\text {comb }}$ at any phase shift. This illustrates that, for $n \neq 1$, the linearity fails to work when $h^{\prime}$ and $u_{s}^{\prime}$ are non-zero. Non-uniformity in either the channel height or the wall charge distribution will trigger pressure to be generated internally; such an induced pressure is needed in order to maintain continuity of flow along the channel. As is well known, the flow rate varies nonlinearly with the pressure gradient for a power-law fluid. Here, the pressure gradient is partly due to the hydrodynamic forcing, and partly due to the electric forcing. This explains why the relationship between the flow rate and the two forcings is nonlinear when the fluid is non-Newtonian and the channel is axially non-uniform. We may infer from Fig. 2 that $q_{\mathrm{PO}}+q_{\mathrm{EO}}<q_{\mathrm{comb}}$ for $n<1$ (shear-thinning fluid), and the opposite is true for $n>1$ (shear-thickening fluid).

The wall undulation will interact with the wall charge modulation differently depending on the phase shift. From Fig. 2, one can see that, for any $n$, the flow rate is the maximum positive when $\phi=\pi$, and is the maximum negative when $\phi=0$. These phases correspond 
respectively to the cases where the maximum positive/negative slip velocity occurs at the narrowest section of the channel. In other words, it is the electric forcing at the narrowest part of the channel that has the most influence on the net flow. This is consistent with the well-known fact that, for flow through a channel with transverse topographical patterns, the flow is rate limited by the smallest cross-section of the channel. An analytical evidence is available from Eq. (23) for the Newtonian fluid flow. For given wall shape and HS slip modulation, they will interact with each other to give rise to the maximum positive effect on the flow when $-h^{\prime} u_{s}^{\prime} \cos \phi$ is the maximum positive, implying that $\phi=\pi$ if $h^{\prime} u_{s}^{\prime}>0$, or $\phi=0$ if $h^{\prime} u_{s}^{\prime}<0$. This statement appears to be true even for $n \neq 1$.

We next show in Fig. 3 the various flow rates as a function of $h^{\prime}$, for $n=0.5,1,1.5$, $\Delta P=1, \bar{u}_{s}=1, u_{s}^{\prime}=0$ (i.e, uniformly charged walls), and $\phi=0$. Again, we see that $q_{\mathrm{PO}}+q_{\mathrm{EO}}=q_{\mathrm{comb}}$ for $n=1$. For $n \neq 1, q_{\mathrm{PO}}+q_{\mathrm{EO}} \neq q_{\mathrm{comb}}$ except in the limiting case of $h^{\prime}=0$ (i.e., flat walls). This confirms our earlier statement that linearity works for a uniform channel $\left(h^{\prime}=u_{s}^{\prime}=0\right)$ for any $n$. Here, Fig. 3 reveals that, even if the walls are uniformly charged, non-uniformity in channel height $\left(h^{\prime} \neq 0\right)$ alone will result in nonlinearity if $n \neq 1$. Again, it is seen that $q_{\mathrm{PO}}+q_{\mathrm{EO}}<q_{\mathrm{comb}}$ for $n<1$, and $q_{\mathrm{PO}}+q_{\mathrm{EO}}>q_{\mathrm{comb}}$ for $n>1$.

For flat walls $\left(h^{\prime}=0\right)$, the effect of $u_{s}^{\prime}$ on the flow rate is shown in Fig. 4 , where $\bar{u}_{s}=1$ and $\phi=0$. In the absence of wall undulation, the periodic variation of the HS slip has no net effect on the flow of a Newtonian fluid, irrespective of the pressure forcing. For nonNewtonian fluid, the response to the pressure forcing is very different. As has been pointed out in Eq. (25), when the walls are flat and there is no applied pressure forcing $(\Delta P=0)$, the flow rate is always unaffected by $u_{s}^{\prime}$ for any $n$. In sharp contrast, in the presence of an applied pressure forcing $(\Delta P \neq 0)$, the flow rate can be appreciably changed by $u_{s}^{\prime}$ for $n \neq 1$. Figure 4 shows that, under a positive $\Delta P, q$ will increase with $u_{s}^{\prime}$ for $n<1$ (shear-thinning fluid), but will decrease with $u_{s}^{\prime}$ for $n>1$ (shear-thickening fluid). This demonstrates that, even without wall undulation, non-uniformity in the HS slip alone may interact nonlinearly with the hydrodynamic forcing for a non-Newtonian fluid.

As has been shown in Fig. 3, $q$ will decrease monotonically with increasing $h^{\prime}$ when $u_{s}^{\prime}=0$. This is no longer the case when $u_{s}^{\prime} \neq 0$. We show in Fig. 5 how $q$ may vary non-monotonically with $h^{\prime}$ for $\Delta P=1, \bar{u}_{s}=0, u_{s}^{\prime}=1$ and $\phi=0$. The non-monotonic behavior arises 
from two competing effects. As $h^{\prime}$ increases in magnitude, the area of the narrowest section decreases (thereby decreasing the flow), but on the other hand the interaction between the wall undulation and the slip modulation will become stronger (thereby enhancing the flow). At the limits $h^{\prime}= \pm 1, q=0$ as the channel height drops to zero at the narrowest section. At $h^{\prime}=0$, the correlation term $h^{\prime} u_{s}^{\prime}$ vanishes, and the resulting flow rate is not the maximum. In fact, the maximum flow rate $q_{\max }$ occurs at a negative value of $h^{\prime}$ in this case. It is because, as remarked above, the association of $h^{\prime}$ and $u_{s}^{\prime}$ will have a positive effect on $q$ only when $-h^{\prime} u_{s}^{\prime} \cos \phi$ is positive. Figure 5 shows that $q$ is always positive when $h^{\prime}$ is negative, but can be negative when $h^{\prime}$ is positive.

It is of practical interest to find out how the maximum flow rate $q_{\max }$ and the corresponding optimum undulation amplitude $h_{\mathrm{optm}}^{\prime}$ may change with the power-law index $n$. Figure 6 shows such relationships for $\Delta P=0,1, \bar{u}_{s}=0, u_{s}^{\prime}=1$ and $\phi=0$. While $q_{\max }$ always decreases with increasing $n, h_{\mathrm{optm}}^{\prime}$ may increase or decrease in magnitude with increasing $n$ depending on $\Delta P$. Clearly, the change of $q_{\max }$ and $h_{\mathrm{optm}}^{\prime}$ with $n$ is more dramatic when $\Delta P$ is non-zero than when it is zero. In the absence of the hydrodynamic forcing, the flow rate is rather insensitive to $n$, even when $n$ varies widely from 0.5 to 1.5 . In sharp contrast, with a hydrodynamic forcing, the maximum flow rate may change appreciably as the power-law index changes. This demonstrates again the nonlinear interaction between the hydrodynamic forcing and the electric forcing in driving the flow of a non-Newtonian fluid through a non-uniform channel.

\section{Concluding remarks}

We have developed a simplified analytical model to describe electrokinetic flow of a power-law fluid through a slit channel with gradually varying wall potential and channel height. The Helmholtz-Smoluchowski (HS) slip condition, which represents a thin Newtonian depletion layer enclosing a still thinner electric double layer, is adopted in this study. We have shown how the wall undulation $\left(h^{\prime}\right)$ and the surface charge modulation $\left(u_{s}^{\prime}\right)$ may interact with each other, when simultaneously driven by pressure and electric forces, to give rise to various nonlinear flow behaviors. Some key findings are summarized as follows. 
1. The Newtonian depletion effect is to cause the flow rate to be insensitive to the powerlaw index when the applied pressure gradient is zero. In the presence of an applied pressure gradient, the power-law rheology becomes more influential on the flow, despite the Newtonian depletion layer.

2. For a non-Newtonian bulk fluid, the flow is a linear combination of the components due separately to the hydrodynamic and electric forcings only when the channel is strictly uniform, or when the flow is strictly one-dimensional.

3. Non-uniformity in either the wall charge or the channel height will induce pressure to be generated internally, which will upset the linearity of the relationship between flow and applied electric field for a non-Newtonian fluid.

4. For a Newtonian bulk fluid, the wall undulation interacts with the charge modulation through the term $-h^{\prime} u_{s}^{\prime} \cos \phi$. For given $h^{\prime}$ and $u_{s}^{\prime}$, the interaction has the maximum positive effect on the flow when the phase $\phi=\pi$ if $h^{\prime} u_{s}^{\prime}>0$, or $\phi=0$ if $h^{\prime} u_{s}^{\prime}<0$. This phase effect appears to apply to non-Newtonian fluids as well.

5. In the absence of wall undulation $\left(h^{\prime}=0\right)$, the periodic change of the HS slip has no effect on the flow rate when the applied pressure gradient is zero, whether the fluid is Newtonian or not. In contrast, when the applied pressure gradient is non-zero, even without the wall undulation, the periodic change of the wall potential will have disparate effects on the flow rate, depending on whether the fluid is shear thinning, Newtonian, or shear thickening.

6. In the absence of wall charge modulation $\left(u_{s}^{\prime}=0\right)$, the flow rate decreases monotonically with increasing amplitude $\left(h^{\prime}\right)$ of the wall undulation. In contrast, when $u_{s}^{\prime} \neq 0$, there exists an optimum amplitude $h_{\text {optm }}^{\prime}$ at which the flow rate is the maximum. This maximum flow rate $q_{\max }$ should happen where $-h_{\mathrm{optm}}^{\prime} u_{s}^{\prime} \cos \phi$ is positive. The maximum flow rate $q_{\max }$ and the optimum undulation amplitude $h_{\mathrm{optm}}^{\prime}$ may vary appreciably with the power-law index $n$ in the presence of an applied pressure gradient. 


\section{Acknowledgments}

Financial support was given by the Research Grants Council of the Hong Kong Special Administrative Region, China, through Project No. HKU 715510E.

\section{References}

[1] S. Chakraborty, Electroosmotically driven capillary transport of typical non-Newtonian biofluids in rectangular microchannels, Anal. Chim. Acta 605 (2007) 175-184.

[2] C.L.A. Berli, M.L. Olivares, Electrokinetic flow of non-Newtonian fluids in microchannels, J. Colloid Interface Sci. 320 (2008) 582-589.

[3] C. Zhao, E. Zholkovskij, J. Masliyah, C. Yang, Analysis of electroosmotic flow of powerlaw fluids in a slit microchannel, J. Colloid Interface Sci. 326 (2008) 503-510.

[4] R.P. Bharti, D.J.E. Harvie, M.R. Davidson, Electroviscous effects in steady fully developed flow of a power-law liquid through a cylindrical microchannel, Int. J. Heat Fluid Flow 30 (2009) 804-811.

[5] M.L. Olivares, L. Vera-Candioti, C.L.A. Berli, The EOF of polymer solutions, Electrophoresis 30 (2009) 921-929.

[6] G.H. Tang, X.F. Li, Y.L. He, W.Q. Tao, Electroosmotic flow of non-Newtonian fluid in microchannels, J. Non-Newtonian Fluid Mech. 157 (2009) 133-137.

[7] C. Zhao, C. Yang, Analysis of power-law fluid flow in a microchannel with electrokinetic effects, Int. J. Emerg. Multi. Fluid Sci. 1 (2009) 37-52.

[8] C. Zhao, C. Yang, Nonlinear Smoluchowski velocity for electroosmosis of power-law fluids over a surface with arbitrary zeta potentials, Electrophoresis 31 (2010) 973-979.

[9] C. Zhao, C. Yang, An exact solution for electroosmosis of non-Newtonian fluids in microchannels, J. Non-Newtonian Fluid Mech. 166 (2011) 1076-1079. 
[10] C.L.A. Berli, Output pressure and efficiency of electrokinetic pumping of nonNewtonian fluids, Microfluid. Nanofluid. 8 (2010) 197-207.

[11] N. Vasu, S. De, Electroviscous effects in purely pressure driven flow and stationary plane analysis in electroosmotic flow of power-law fluids in a slit microchannel, Int. J. Eng. Sci. 48 (2010) 1641-1658.

[12] N. Vasu, S. De, Electroosmotic flow of power-law fluids at high zeta potentials, Colloids and Surfaces A: Physicochem. Eng. Aspects 368 (2010) 44-52.

[13] A. Babaie, A. Sadeghi, M.H. Saidi, Combined electroosmotically and pressure driven flow of power-law fluids in a slit microchannel, J. Non-Newtonian Fluid Mech. 166 (2011) 792-798.

[14] M. Hadigol, R. Nosrati, M. Raisee, Numerical analysis of mixed electroosmotic/pressure driven flow of power-law fluids in microchannels and micropumps, Colloids and Surfaces A: Physicochem. Eng. Aspects 374 (2011) 142-153.

[15] A. Sadeghi, M. Fattahi, M.H. Saidi, An approximate analytical solution for electroosmotic flow of power-law fluids in a planar microchannel, J. Heat Trans. 133 (2011) 091701.

[16] C.C. Cho, C.L. Chen, C.K. Chen, Electrokinetically-driven non-Newtonian fluid flow in rough microchannel with complex-wavy surface, J. Non-Newtonian Fluid Mech. 173174 (2012) 13-20.

[17] C.C. Cho, C.L. Chen, C.K. Chen, Flow characteristics and mixing performance of electrokinetically driven non-Newtonian fluid in contraction-expansion microchannel, Rheol. Acta 51 (2012) 925-935.

[18] S.Y. Deng, Y.J. Jian, Y.H. Bi, L. Chang, H.J. Wang, Q.S. Liu, Unsteady electroosmotic flow of power-law fluid in a rectangular microchannel, Mech. Res. Comm. 39 (2012) 914.

[19] M. Shamshiri, R. Khazaeli, M. Ashrafizaadeh, S. Mortazavi, Electroviscous and thermal effects on non-Newtonian liquid flows through microchannels, J. Non-Newtonian Fluid Mech. 173-174 (2012) 1-12. 
[20] M.A. Vakili, A. Sadeghi, M.H. Saidi, A.A. Mozafari, Electrokinetically driven fluidic transport of power-law fluids in rectangular microchannels, Colloids and Surfaces A: Physicochem. Eng. Aspects 414 (2012) 440-456.

[21] Q. Zhu, S. Deng, Y. Chen, Periodical pressure-driven electrokinetic flow of power-law fluids through a rectangular microchannel, J. Non-Newtonian Fluid Mech. 203 (2014) 38-50.

[22] S. Das, S. Chakraborty, Analytical solutions for velocity, temperature and concentration distribution in electroosmotic microchannel flows of a non-Newtonian bio-fluid, Anal. Chim. Acta 559 (2006) 15-24.

[23] C.O. Ng, C. Qi, Electroosmotic flow of a viscoplastic material through a slit channel with walls of arbitrary zeta potential, Phys. Fluids 25 (2013) 103102.

[24] H.A. Barnes, A review of the slip (wall depletion) of polymer solutions, emulsions and particle suspensions in viscometers: its cause, character, and cure, J. Non-Newtonian Fluid Mech. 56 (1995) 221-251.

[25] R. Tuinier, T. Taniguchi, Polymer depletion-induced slip near an interface, J. Phys.: Condens. Matter 17 (2005) L9-L14.

[26] W.B. Zimmerman, J.M. Rees, T.J. Craven, Rheometry of non-Newtonian electrokinetic flow in a microchannel T-junction, Microfluid. Nanofluid. 2 (2006) 481-492.

[27] A. Ajdari, Generation of transverse fluid currents and forces by an electric field: electroosmosis on charge-modulated and undulated surfaces, Phys. Rev. E 53 (1996) 49965005.

[28] A. Ajdari, Transverse electrokinetic and microfluidic effects in micropatterned channels: lubrication analysis for slab geometries, Phys. Rev. E 65 (2001) 016301.

[29] D. Long, H.A. Stone, A. Ajdari, Electroosmotic flows created by surface defects in capillary electrophoresis, J. Colloid Interf. Sci. 212 (1999) 338-349.

[30] S. Ghosal, Lubrication theory for electro-osmotic flow in a microfluidic channel of slowly varying cross-section and wall charge, J. Fluid Mech. 459 (2002) 103-128. 
[31] C.O. Ng, Q. Zhou, Electro-osmotic flow through a thin channel with gradually varying wall potential and hydrodynamic slippage, Fluid Dyn. Res. 44 (2012) 055507.

[32] C.O. Ng, Q. Zhou, Dispersion due to electroosmotic flow in a circular microchannel with slowly varying wall potential and hydrodynamic slippage, Phys. Fluids 24 (2012) 112002.

[33] J.M. MacInnes, X. Du, R.W.K. Allen, Prediction of electrokinetic and pressure flow in microchannel T-junction, Phys. Fluids 15 (2003), 1992-2005.

[34] J.M. MacInnes, Computation of reacting electrokinetic flow in microchannel geometries, Chem. Eng. Sci. 57 (2002) 4539-4558. 


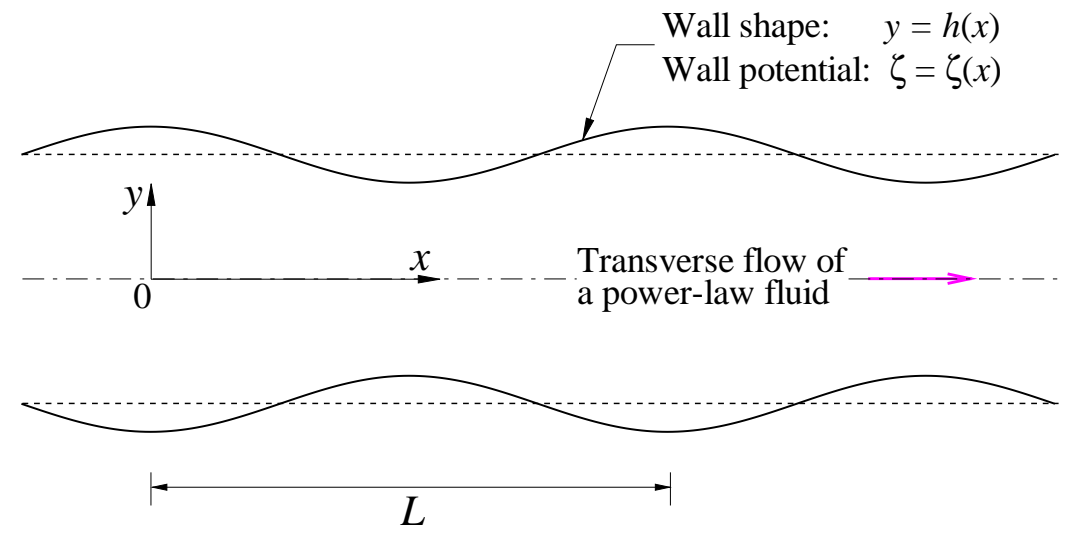

Figure 1: Transverse pressure-driven and electroosmotic flow of a power-law fluid through a slit channel with undulated walls and charge-modulated surfaces. 

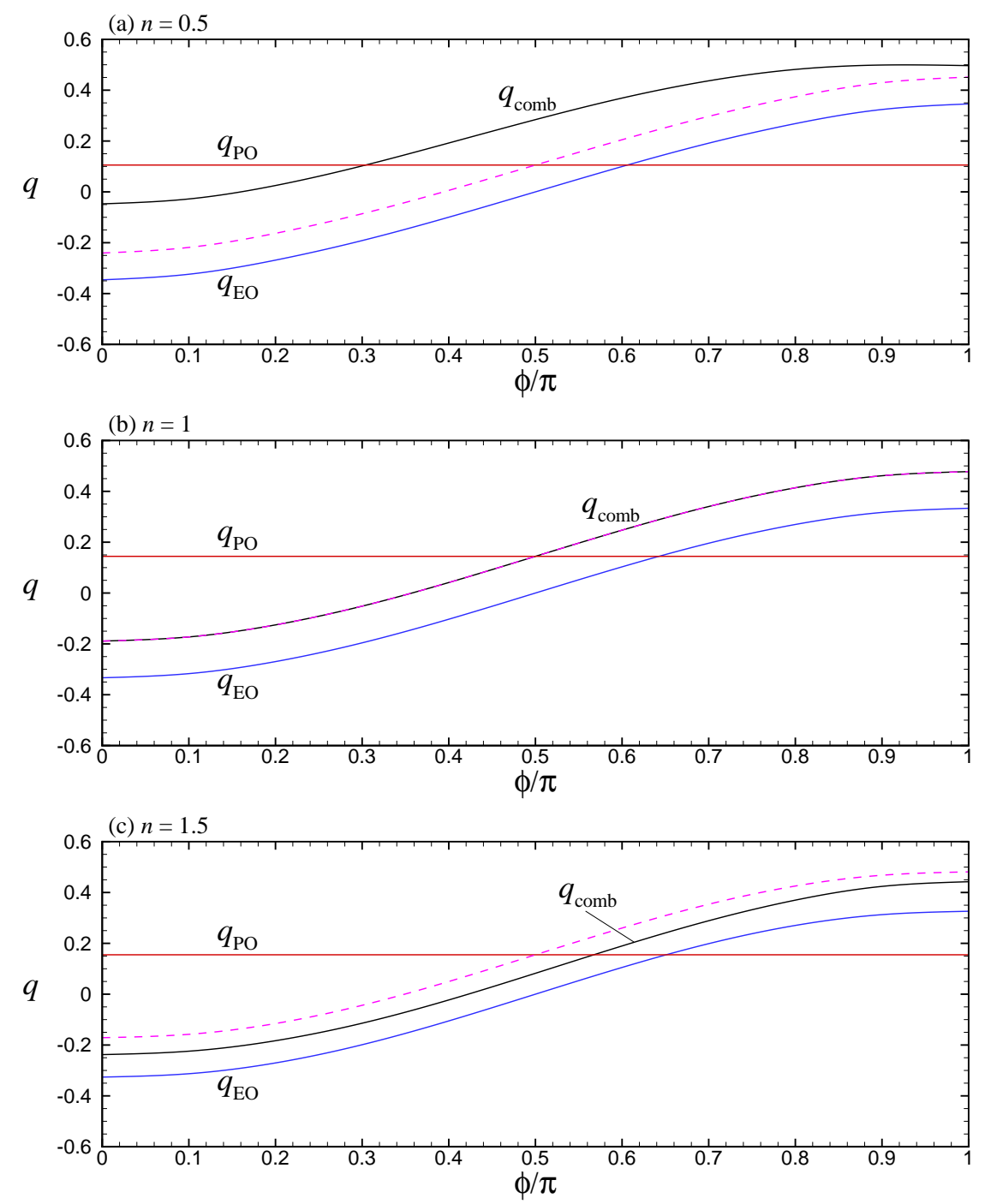

Figure 2: Flow rate $q$ as a function of the phase $\phi$ for $\Delta P=1, h^{\prime}=0.5, \bar{u}_{s}=0, u_{s}^{\prime}=1$, and (a) $n=0.5$, (b) $n=1$, (c) $n=1.5$, where $q_{\mathrm{PO}}, q_{\mathrm{EO}}, q_{\mathrm{comb}}$ are the flow rates defined in Eqs. (26)-(28), and the dashed line represents $q_{\mathrm{PO}}+q_{\mathrm{EO}}$. For $n=1, q_{\mathrm{comb}}=q_{\mathrm{PO}}+q_{\mathrm{EO}}$. 

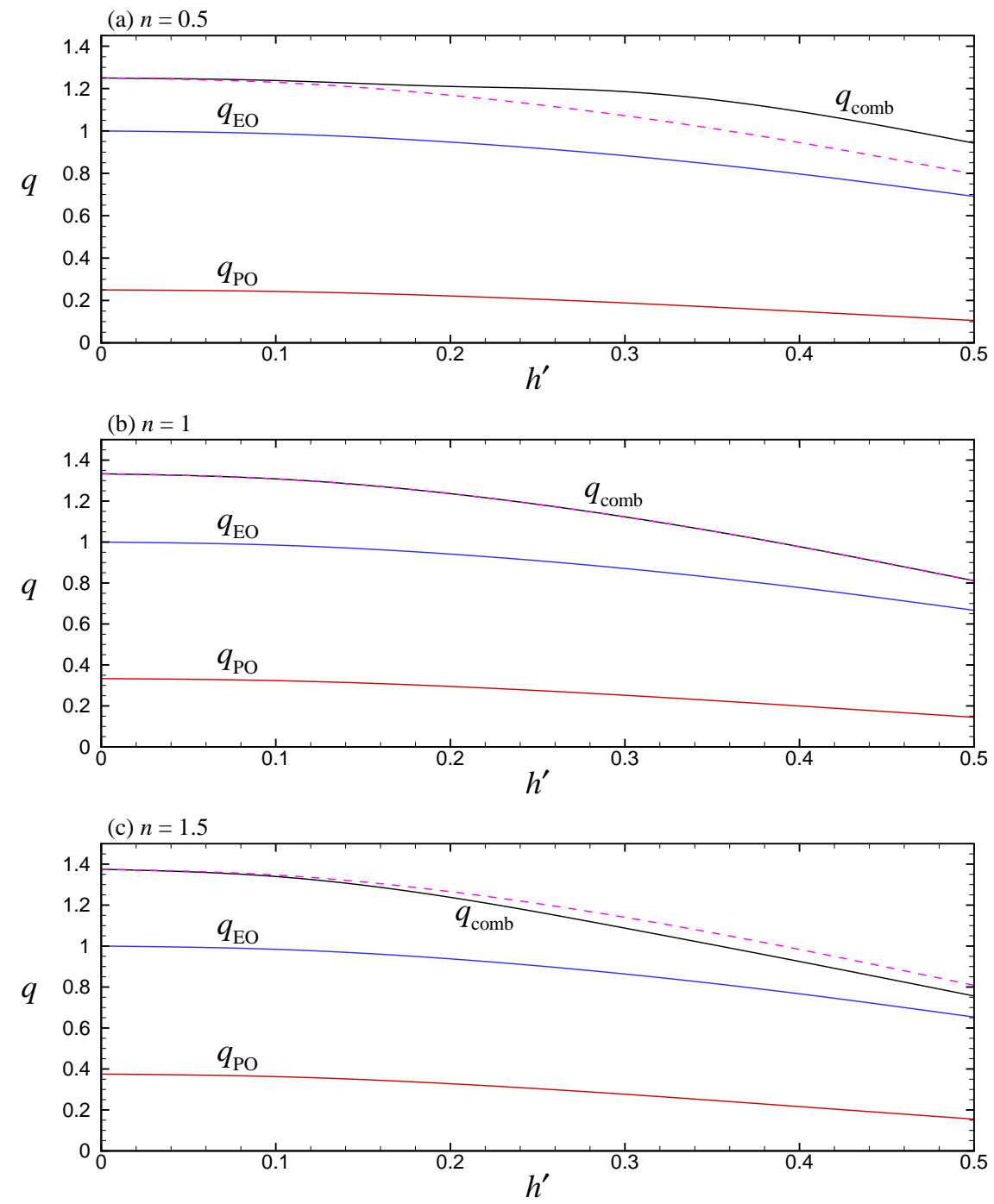

Figure 3: Flow rate $q$ as a function of the wall undulation amplitude $h^{\prime}$ for $\Delta P=1, \bar{u}_{s}=1$, $u_{s}^{\prime}=0, \phi=0$, and (a) $n=0.5$, (b) $n=1$, (c) $n=1.5$, where $q_{\mathrm{PO}}, q_{\mathrm{EO}}, q_{\mathrm{comb}}$ are the flow rates defined in Eqs. (26)-(28), and the dashed line represents $q_{\mathrm{PO}}+q_{\mathrm{EO}}$. For $n=1$, $q_{\mathrm{comb}}=q_{\mathrm{PO}}+q_{\mathrm{EO}}$. 

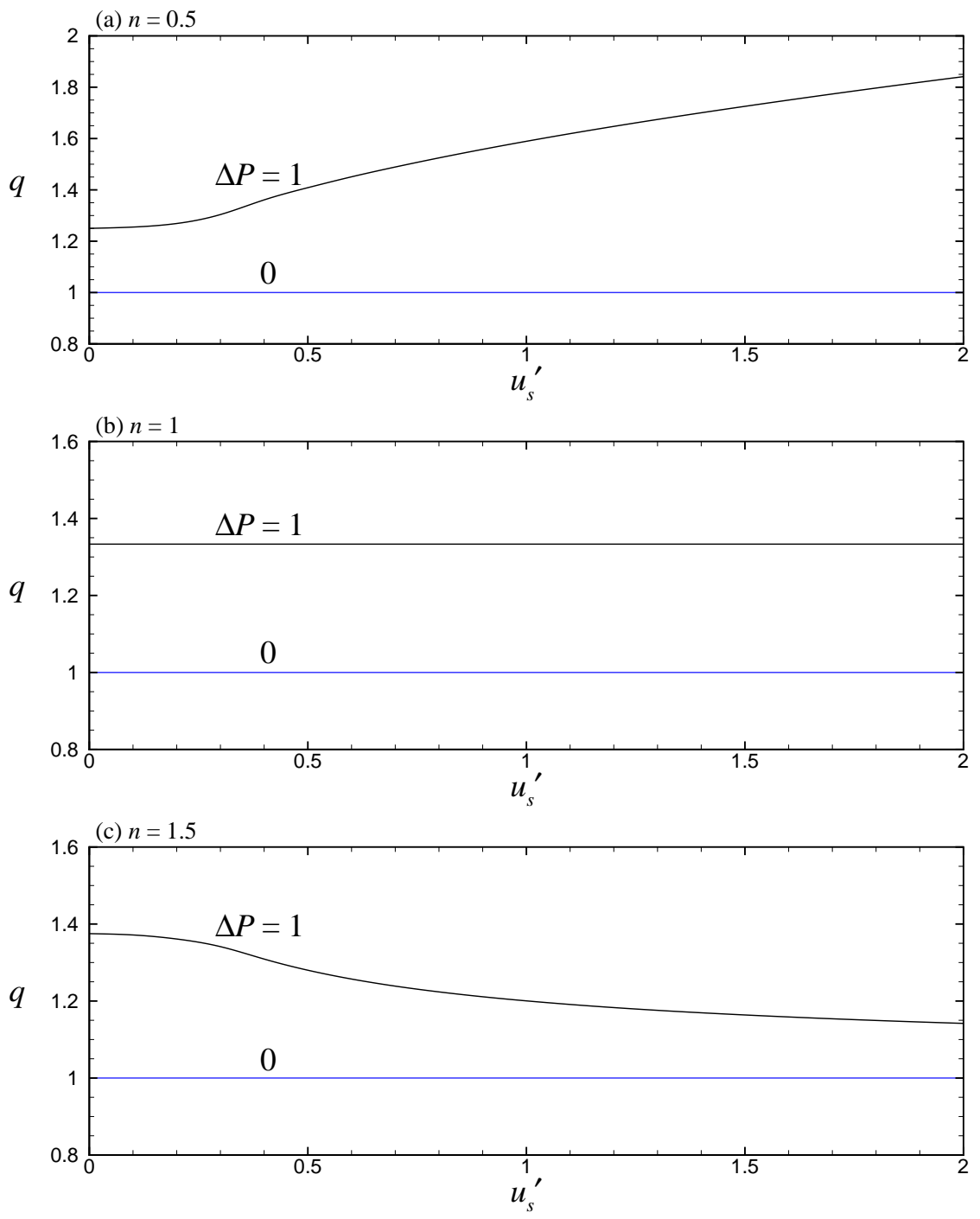

Figure 4: Flow rate $q$ as a function of the HS slip modulation amplitude $u_{s}^{\prime}$ for $\Delta P=1,0$, $h^{\prime}=0, \bar{u}_{s}=1, \phi=0$, and (a) $n=0.5$, (b) $n=1$, (c) $n=1.5$. 


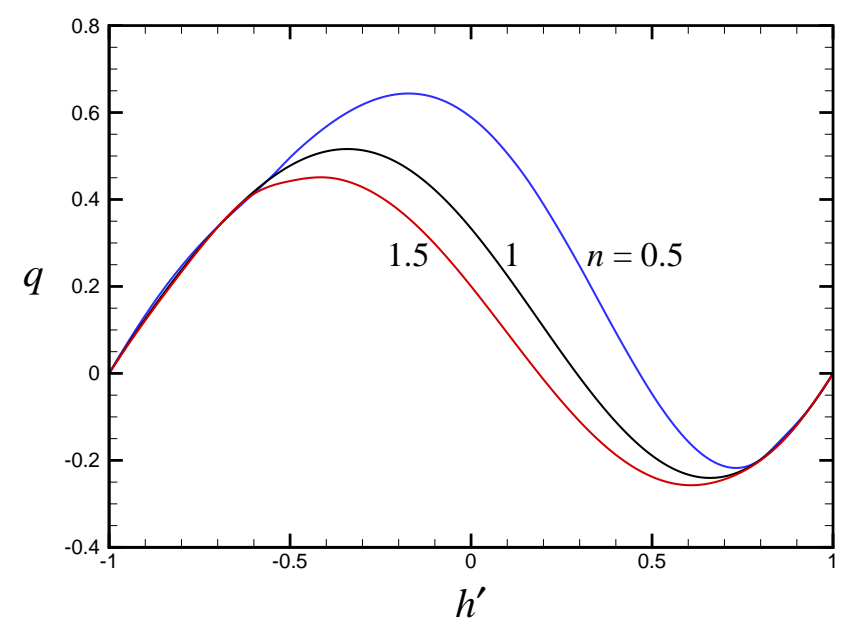

Figure 5: Flow rate $q$ as a function of the wall undulation amplitude $h^{\prime}$ for $\Delta P=1, \bar{u}_{s}=0$, $u_{s}^{\prime}=1, \phi=0$, and $n=0.5,1,1.5$. 

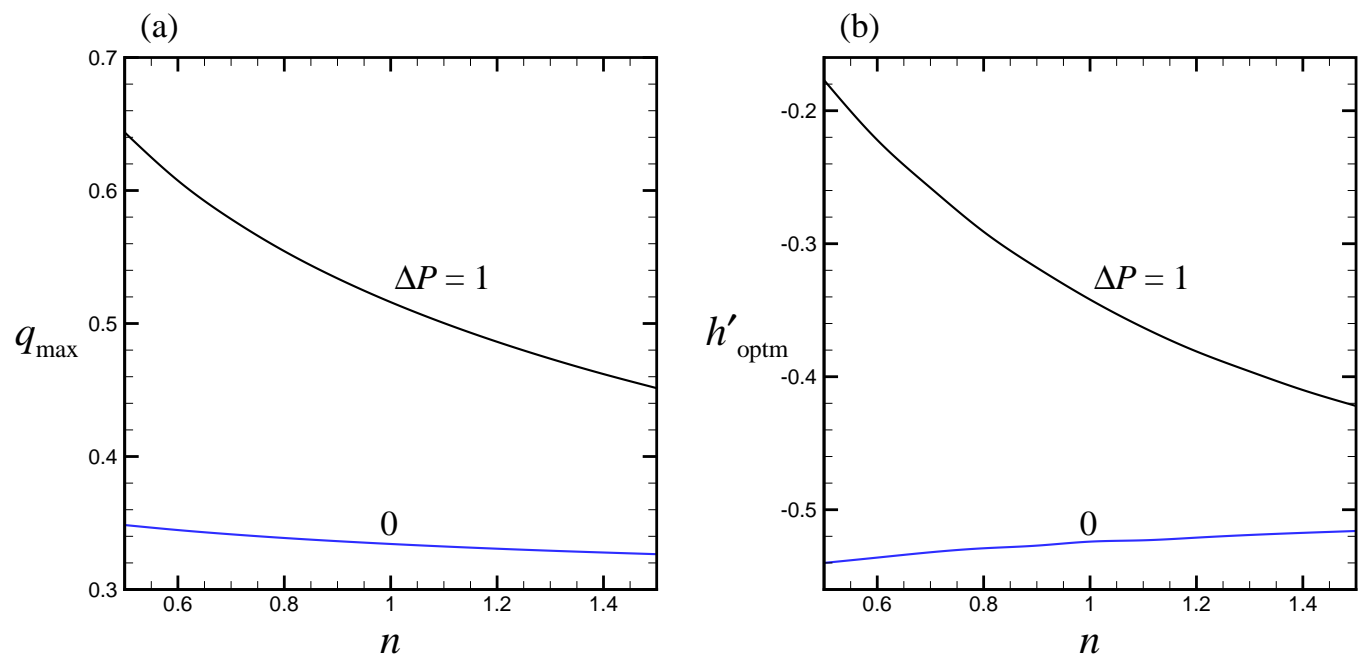

Figure 6: Maximum flow rate $q_{\max }$ and the corresponding optimum wall undulation amplitude $h_{\mathrm{optm}}^{\prime}$ as a function of the power-law index $n$, for $\Delta P=1,0, \bar{u}_{s}=0, u_{s}^{\prime}=1$ and $\phi=0$. 